\title{
Dietary thiols in exercise: oxidative stress defence, exercise performance, and adaptation
}

\author{
Yanita McLeay ${ }^{1 *} \mathbb{D}$, Stephen Stannard ${ }^{1}$, Stuart Houltham ${ }^{1}$ and Carlene Starck ${ }^{2}$
}

\begin{abstract}
Endurance athletes are susceptible to cellular damage initiated by excessive levels of aerobic exercise-produced reactive oxygen species (ROS). Whilst ROS can contribute to the onset of fatigue, there is increasing evidence that they play a crucial role in exercise adaptations. The use of antioxidant supplements such as vitamin $C$ and $E$ in athletes is common; however, their ability to enhance performance and facilitate recovery is controversial, with many studies suggesting a blunting of training adaptations with supplementation. The up-regulation of endogenous antioxidant systems brought about by exercise training allows for greater tolerance to subsequent ROS, thus, athletes may benefit from increasing these systems through dietary thiol donors. Recent work has shown supplementation with a cysteine donor (N-acetylcysteine; NAC) improves antioxidant capacity by augmenting glutathione levels and reducing markers of oxidative stress, as well as ergogenic potential through association with delayed fatigue in numerous experimental models. However, the use of this, and other thiol donors may have adverse physiological effects. A recent discovery for the use of a thiol donor food source, keratin, to potentially enhance endogenous antioxidants may have important implications for endurance athletes hoping to enhance performance and recovery without blunting training adaptations.
\end{abstract}

Keywords: Endurance, Thiol, Antioxidant

\section{Background}

It is well-established that muscular work is associated with oxidative stress and that prolonged or high-intensity exercise results in oxidative damage to macromolecules in both blood and skeletal muscle [1-3]. This exerciseinduced oxidative damage can impair physical performance via various mechanisms relating to compromised myocellular structure and function. Furthermore, chronic oxidative stress in athletes, often brought about by overtraining, has been linked to chronic fatigue [4], longer term performance decrements [5], muscle atrophy [6], and illness [7]. Exogenous and endogenous antioxidants reduce oxidative stress, thus it is not surprising that dietary antioxidants are a popular supplement for athletes in an attempt to enhance recovery, optimise performance, and reduce the oxidant load to maintain long-term health.

\footnotetext{
* Correspondence: y.mcleay@massey.ac.nz

'School of Sport and Exercise, Massey University, Private Bag 11-222,

Palmerston North, New Zealand

Full list of author information is available at the end of the article
}

The two groups of biologically active molecules that fall under the umbrella term 'free radicals' include reactive oxygen species (ROS) and reactive nitrogen species (RNS). During exercise, oxygen consumption can increase as much as 100-fold [8] and is associated with rapid increases in ROS production and accumulation. This constant production of free radicals by skeletal muscle requires the buffering capacity of an endogenous defense system, and a multitude of mechanisms have evolved to detect and respond to elevated oxidant production. The action of these systems determines the overall endogenous antioxidant capacity, and if this capacity is exceeded, oxidative stress ensues, potentially resulting in detrimental oxidation of cell membranes, functional proteins, and DNA [9-11].

Paradoxically, the redox activities of ROS and RNS play critical roles in cell signaling and exercise adaptation, a phenomenon summarized by the concept of hormesis, where low levels of stress promote adaptation to and thus protection from, subsequent stress [12]. Various exercise studies have observed improved endogenous defence in 
rodents following training [13-17], along with reduced ROS production at the same absolute exercise workload during following training [18]. However, at very high concentrations, ROS can move beyond being advantageous, and have detrimental effects on performance. During heavy endurance training, endogenous antioxidant capacity cannot meet the increasingly high ROS generation, resulting in a state of oxidative stress and subsequent cellular damage [19]. Dietary supplementation with antioxidants in this situation may be beneficial; however, the exact 'balance' of (exogenous) antioxidant supplementation to ROS levels that may enhance performance without blunting adaptive pathways is currently unknown. Commonly supplemented antioxidants include the well-known vitamins such as $C$ and $E$, and in recent times, research concerning the effects of these on exercise performance in humans has grown considerably, producing equivocal results [20-24]. While acute administration in humans may potentially enhance performance [25], the majority of studies suggest no benefit [23, 26-30]. Furthermore, there is concern that chronic supplementation may blunt the free radical-driven adaptive effects of training by interfering with the essential endogenous antioxidant response to ROS [31, 32]. More recently, the thiol donors' glutathione, cysteine, and taurine, have generated interest for the improvement of exercise recovery and performance, with their potential to boost endogenous antioxidant defence in a way that generalized antioxidants cannot. However, the use of these is limited by poor supplement bioavailability and several side effects [33, 34]. This has created a niche for the advent of palatable, digestible, and tolerated dietary thiol sources. In this review we focus on the emergence of thiols as antioxidant supplements for the endurance athlete, the current research surrounding their ergogenic potential and benefits, and recent efforts to produce a high-cysteine food source.

\section{Antioxidant supplementation, exercise performance, and adaptation}

While research into the effect of common dietary antioxidants on acutely reducing free-radical load is well documented in humans, evidence of any exercise performance or adaptation benefits are equivocal [20-23, 30, 31, 35-38]. Few studies have observed performance benefits as a result of supplementing with specific dietary antioxidants [37] and many well controlled studies in this area have found no performance benefit [21-23, 39-42]. It has been suggested that antioxidant supplementation may only improve performance when endogenous levels are already depleted, and after reaching normal concentrations, no further benefit is seen [35]. Similarly, antioxidant supplementation does not appear to benefit recovery time following acute exercise [43-46], with only a limited number of studies showing minor recovery benefits [30, 47]. On the other hand, there is an increasing body of work that has shown that ingestion of specific foods, high in antioxidant compounds can accelerate recovery $[44,48,49]$. Both cherries $[44,48]$ and blueberries [49] seem to expedite the recovery of muscular force following strenuous eccentric work. However, whether this is due to the antioxidant capabilities of the foods, or the polyphenolics is a point of contention. Our previous work [49] seems to infer the latter.

A number of published studies to date indicate that supplementation with common dietary antioxidants may actually be detrimental to athletic performance by blunting the adaptive effect of exercise training [31, 50-52]. These adaptations, including upregulated endogenous defense, muscle protein synthesis, and mitochondrial biogenesis, are important for improved exercise capacity and recovery. Dietary antioxidants such as vitamins $\mathrm{C}$ and $E$ are generally required in very small amounts, working with endogenous antioxidants to maintain or re-establish redox homeostasis [53]. However, these general antioxidants non-specifically scavenge all free radicals, regardless of their source; hence increased intake may affect various pathways including cellular signaling pathways that are important for exercise adaptation. In contrast, endogenous antioxidant systems are a complex and compartmentalized network that involve controlled and localized production of specific ROS [54]. These ROS are uniquely balanced by endogenous antioxidants to levels where cellular signaling can still be carried out. In athletes, disruption of this system by over-supplementation with generalized exogenous antioxidants can result in blunted signaling pathways $[31,38,50]$. This in turn can compromise recovery and attenuate exercise adaptations, negatively affecting performance.

In addition to their effect on adaptive pathways, at high levels, exogenous antioxidants can promote oxidation, contributing to acute decrements in exercise performance. For example, vitamin $\mathrm{C}$ can react with metal ions released from exercise-induced tissue damage, giving rise to harmful hydroxyl radicals [55], and vitamin E can become a free radical itself when it reacts with a free radical [56], damaging lipid membranes if it is not converted back to its reduced form. Following acute eccentric induced muscle injury in young men, Childs et al. [57] found increased oxidative stress and tissue damage supplementation with vitamin $\mathrm{C}$ and a cysteine derivative $\mathrm{n}$-acytylcysteine (NAC), compared to a placebo. Similarly, in ultraendurance (Ironman ${ }^{\mathrm{Tx}}$ ) competitors, vitamin $\mathrm{C}$ or $\mathrm{E}$ supplementation prior to racing significantly elevated markers of oxidative stress and showed significant decreases in antioxidant enzyme activity post- race, compared with no supplementation [58]. For a more comprehensive review on the various dietary antioxidants that can form a prooxidant state, please refer to Yordi et al. [59]. 
In contrast to exogenous antioxidants, high levels of endogenous antioxidants do not appear to contribute to oxidative stress. Rather, it is depleted levels that can give rise to an oxidant state [60]. It could therefore be suggested that consuming dietary sources of endogenous antioxidant precursors, such as thiol donors, may enhance exercise performance and recovery without the negative effects associated with exogenous antioxidant supplementation.

\section{Thiols}

\section{Antioxidant capacity}

Thiols, molecules that contain a sulfhydyl (SH) side chain group, act as antioxidants, stabilizing free radicals by accepting their unpaired electron. Methionine and cysteine are two key dietary thiol amino acids that metabolize to the powerful and arguably most important endogenous antioxidant, glutathione (GSH). Adequate levels of GSH are crucial in maintaining reductionoxidation (redox) balance within body tissues with the ratio of reduced to oxidized glutathione (GSH/GSSG) being a primary indicator of redox state. A higher ratio of GSH to GSSG suggests a reductive environment where ROS levels are kept at homeostatic levels, whereas a low GSH to GSSG ratio is indicative of oxidative stress [61]. It is cysteine that gives GSH its antioxidant activity; as cysteine is also rate limiting to its formation, dietary cysteine, or its precursor amino acid methionine, is crucial for maintaining endogenous antioxidant defense. In addition to GSH, dietary thiols have the ability to increase levels of taurine, another powerful thiol antioxidant, via the cysteine sulfinic acid pathway (Fig. 1).

Various studies using thiol donor supplementation have shown positive effects on the up-regulation of endogenous antioxidants and antioxidant enzyme activity. For example, administration of the cysteine-donor $\mathrm{N}$-acetyl cysteine (NAC) in humans significantly increases blood [62-64] and muscle $[65,66]$ GSH levels, and in vitro studies show methionine supplementation to have beneficial effects on GSH hepatocyte concentrations [67]. Furthermore, NAC has been shown to increase taurine levels in skeletal muscle [68].

\section{Exercise performance}

While it appears that excessive intakes of common dietary antioxidants may contribute to oxidative stress [56, 69], there is no published literature to suggest high levels of endogenous thiol antioxidants to have the same effect. Exercise appears to significantly alter GSH and taurine tissue levels, and supplementing with thiol donors has been shown to attenuate this [70]. Reduced markers of oxidative damage have been seen post-exercise with thiol supplementation [71], suggestive of reduced ROS levels. Thus, in contrast to the well-researched vitamins $C$ and $E$, several human studies have suggested that the observed ergogenic effect of thiol donors such as NAC is due to improved endogenous defense systems [72, 73].

Perhaps due to potential toxicity associated with methionine supplementation, to our knowledge no published studies have looked into the use of methionine on

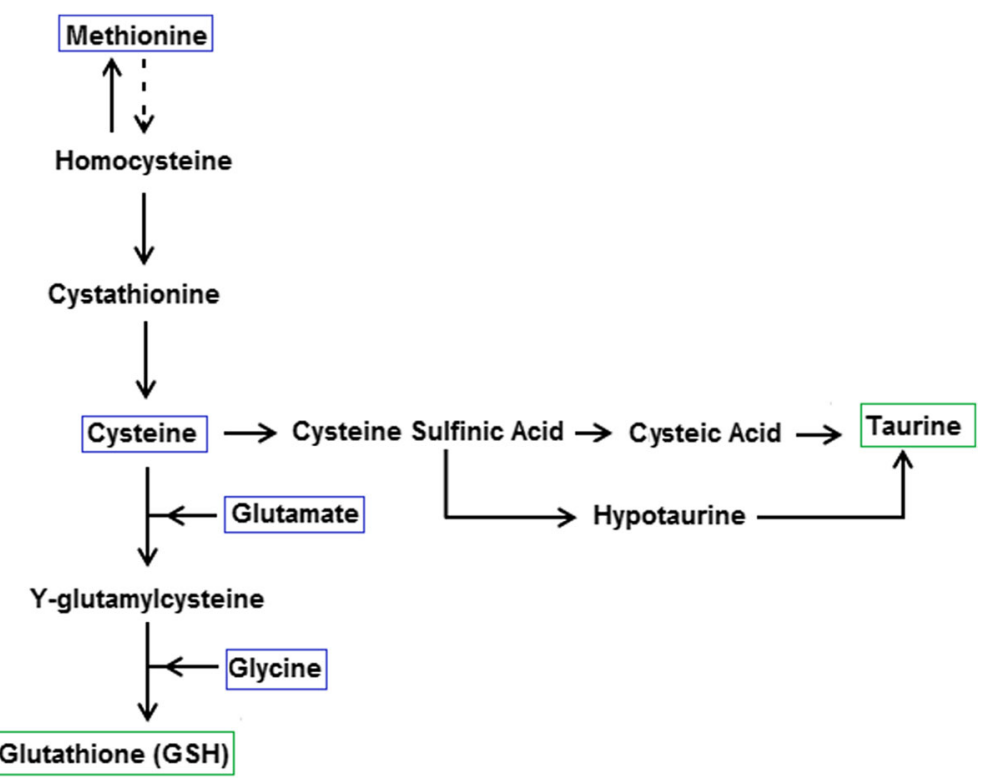

Fig. 1 Summary of thiol metabolism and resulting thiol antioxidants. Methionine is metabolized to cysteine which further forms endogenous antioxidants taurine and glutathione via two distinct pathways. Supplementing with taurine and/or cysteic acid may 'spare' cysteine, upregulating GSH synthesis, thus further boosting endogenous antioxidant defence 
exercise performance in either humans or animals. Similarly, as an isolated supplement, L-cysteine has been shown to elicit toxic effects in several animal studies [74-76]. Because of this, the majority of studies looking at cysteine supplementation on endogenous antioxidant up-regulation use NAC, which endogenously cleaves cystine into two cysteine molecules [77]. Additionally, studies that use exogenous forms of GSH are not common as it is primarily degraded to its constituent amino acids [78]. Exogenous taurine supplementation however, has shown some promising results on increasing time to fatigue during exhaustive exercise by up to $50 \%$ in rodents, and time to $\mathrm{VO}_{2 \max }$ in men [79-81]. Therefore, increasing GSH and taurine levels within the body via thiol donors may benefit athletes.

In rats, NAC infusion at $150 \mathrm{mg} \cdot \mathrm{kg}^{-1}$ was found to increase tolerance to respiratory loading, while also attenuating a decrease in diaphragm GSH [82]. Similarly in humans, the same infusion rate of NAC during electrical stimulation of the ankle dorsiflexors was shown to increase force output by 15\% compared to placebo [83]. Furthermore, Medved and colleagues [65] found that infusing NAC (150 mg. $\left.\mathrm{kg}^{-1}\right)$ into endurance trained men prior to a fatiguing cycling protocol gave rise to a $26.3 \%$ increase in time to exhaustion at $92 \% \mathrm{VO}_{2} \max$ compared to placebo; although in other research from the same group [73], cycling time to fatigue was not affected by an infusion of $125 \mathrm{mg} \cdot \mathrm{kg}^{-1}$, despite an attenuation in the reduction of GSH. In contrast to these studies, Matuszaczak et al. [84] found an infusion of $150 \mathrm{mg} . \mathrm{kg}$ ${ }^{-1}$ to have no effect on force production during sustained maximal handgrip contractions compared to placebo. It did however inhibit GSH oxidation.

A role for taurine has been implicated in virtually every body tissue, with the highest concentrations found within skeletal and cardiac muscle [85]. Maintaining adequate taurine is important for endurance athletes, as taurine also plays crucial roles in muscle contraction and relaxation [86], lipid metabolism [87], and potentially muscle protein synthesis [88]. Furthermore, a possible 'sparing' of GSH has been observed with taurine supplementation [89], suggesting indirect redox-balancing roles. While several earlier studies, using taurine-containing energy drinks, observed a combination of taurine and caffeine to enhance exercise performance above that of caffeine alone [90, 91], it is only more recently that isolated taurine has been used in exercise research. Several studies have shown that two weeks of taurine supplementation in rats elicits 50\% $[81,92]$ and $34 \%$ [79] greater time to exhaustion during treadmill running compared to non-supplemented (control) animals. The impact of taurine supplementation on human exercise performance is less clear. In recreationally trained men, taurine supplementation prior to cycling to $\mathrm{VO}_{2 \max }$ reduced oxidative stress markers in plasma post-cycle, and also significantly increased time to exhaustion at $\mathrm{VO}_{2 \max }$, and maximal workload [80]. Similarly, in trained runners taurine ingestion prior to a $3-\mathrm{km}$ time trial significantly improved performance [93]. In contrast however, Rutherford et al. [94] found acute ingestion of taurine to have no effect on time-trial performance in well-trained cyclists, although it did appear to significantly increase fat oxidation during submaximal cycling at the same absolute work rate.

Overall, the effect of thiol donors on human performance appear to vary, probably due to vast methodological differences including exercise protocol, dosage amount, length of time, and subject training status. However, if the provision of precursors for endogenous antioxidants, such as NAC for GSH and/or taurine, enables the body to regulate to optimal levels its own antioxidant defence mechanism, this may be preferable to the use of general exogenous antioxidants such as vitamins $\mathrm{C}$ and $\mathrm{E}$.

\section{Exercise and adaptation}

In addition to performance, the ability to improve physical capacity over time is of utmost importance for athletes. The human body has the ability to adapt to exercise stressors, allowing for a greater tolerance to a subsequent similar workload, and relies on various ROS-mediated signaling pathways to do so. At low levels, exercise-induced ROS play a critical role in skeletal muscle adaptation [95] by up-regulating various cytokines and protein kinases such as tumor necrosis factor alpha (TNF- $\alpha$ ) [96] and mitogen-activated protein kinases (MAPK) [97] that work to signal increases in mitochondrial density and number, endogenous antioxidants, and assist in muscle protein synthesis [98, 99]. Certain studies have observed upregulated endogenous antioxidants and antioxidant enzymes, specifically superoxide dismutase (SOD) and glutathione peroxidase (GPx) following endurance training $[18,100]$, and well trained athletes appear to have higher base-line levels of these enzymes, as well as GSH [101]. Additionally, while muscle protein breakdown is stimulated during exercise, protein synthesis is increased during recovery through ROS signaling [102]. The use of common antioxidants vitamins $\mathrm{C}$ and $\mathrm{E}$ prior to exercise have been shown to mitigate ROS signaling pathways, resulting in the blunting of such adaptations [31, 36, 50-52].

While exogenous antioxidants may attenuate training adaptations, there is no literature to suggest that increasing endogenous antioxidants has this effect. Rather, a recent study [103] in cyclists undergoing strenuous physical training observed improved physiological adaptation with a week of oral NAC while several other studies [72, 104] have found increased signaling cytokines such as TNF- $\alpha$ with NAC supplementation compared to a placebo, despite improved performance. This suggests that NAC may contribute to exercise adaptation by 
increasing levels of the various signaling molecules. As opposed to oral supplementation however, studies using direct infusion of NAC appear to attenuate the increase in these signaling molecules $[105,106]$. Thus it is important to consider the method of administration when analyzing effect. Whilst NAC appears to upregulate endogenous antioxidants in those who have depleted levels, in healthy individuals, high doses may have prooxidant effects [107-109]. Furthermore, in some individuals, oral [110] and systemically administered [83] NAC can be poorly tolerated. Little research exists on taurine's effect on exercise adaptions; however, taurine appears to have potent cytoprotective roles in skeletal muscle $[88,111]$. Various studies have shown taurine depletion or attenuation of its transport to reduce muscle function and increase atrophy [112]. In addition, several studies have observed a reduction in eccentric-induced muscle damage [113] and oxidative stress [113, 114] following taurine supplementation, suggesting its potential role in recovery via reducing initial tissue damage.

Additional research into the effects of long term use of thiol donors is necessary; however, early observations suggest that they may be useful as ergogenic aids for endurance athletes without attenuating useful adaptations.

\section{A future of thiols as ergogenic aids}

Despite their potential benefits, the use of supplementary thiol donors to improve endogenous antioxidant status may not necessarily be the ideal way to enhance performance and recovery. As previously mentioned, high intakes of NAC in healthy individuals may have pro-oxidant effects [107-109], and both oral [110] and systemically administered [83] NAC can be poorly tolerated. Similarly, while methionine supplementation has been shown to increase GSH levels in those with sub-optimal levels $[115,116]$, excess intakes in healthy individuals may increase homocysteine levels which has been shown to contribute to cardiovascular disease and several mental health disorders $[117,118]$. Therefore, alternative dietary sources of thiols may provide a safer option for athletes and others.

\section{Food-based thiol donors}

Keratin is found in tissues including hair, skin, nails, and feathers, and is comprised of cysteine-based disulfide bonds. While necessary for structural purposes, these strong linkages between cysteine residues render keratin indigestible to humans and thus unavailable for absorption. However, hydrolysed keratin, through either acid or alkaline hydrolysis vastly improves digestibility [119] and sets this protein up as a potential thiol source. High in cysteic acid, a metabolite of cysteine, keratin hydrolysate may directly increase taurine levels; indeed, a recent study in rats [120] observed increased liver taurine following four weeks of keratin supplementation, along with the maintenance of GSH levels, suggestive of a sparing effect. A later study tested the same keratin protein in humans over two weeks using a ramped dose protocol. Starting at $10 \mathrm{~g}$ of keratin/day $\left(10 . \mathrm{g} . \mathrm{d}^{-1}\right)$ for three days, the supplementation period finished with an intake of $40 \mathrm{~g} . \mathrm{d}^{-1}$; a level at which there were still no adverse physiological effects reported [119]. These two studies may have implications for the use of keratinbased thiol supplementation in athletes; however, research into this area is scarce.

\section{Conclusions}

Endurance athletes are susceptible to cellular damage initiated by excessive levels of aerobic exercise-produced ROS. Whilst ROS can contribute to the onset of fatigue, there is increasing evidence that they play a crucial role in exercise adaptations. The use of general antioxidant supplements such as vitamin $C$ and $E$ in athletes is common; however, their ability to enhance performance and facilitate recovery is controversial, with many studies suggesting a blunting of training adaptations with chronic supplementation. However, as the up-regulation of endogenous antioxidant systems are brought about by exercise training, athletes may benefit from increasing these systems through dietary thiol donors. While the thiol donors methionine and NAC may increase endogenous antioxidants and antioxidant enzymes, there can be adverse effects associated with their use. Thus, the discovery for the use of hydrolysed keratin to potentially enhance endogenous GSH and taurine may have important implications for athletes hoping to enhance performance and recovery without blunting training adaptation.

\section{Abbreviations \\ GPx: Glutathione peroxidase; GSH: Glutathione (reduced); GSSG: Glutathione (oxidized); $\mathrm{H}_{2} \mathrm{O}_{2}$ : Hydrogen peroxide; MAPK: Mitogen-activated protein kinases; NAC: N-acetyl cysteine; RNS: Reactive nitrogen species; ROS: Reactive oxygen species; SOD: Superoxide dismutase; TNF-a: Tumor necrosis factor-alpha}

Acknowledgements

Not applicable.

Funding

Not applicable.

Availability of data and materials

Not applicable

Authors' contributions

YM, SS, SH \& CS contributed to this review. All authors read and approved the final manuscript.

Competing interests

The authors declare that they have no competing interests.

Consent for publication

Not applicable.

Ethics approval and consent to participate Not applicable. 


\section{Publisher's Note}

Springer Nature remains neutral with regard to jurisdictional claims in published maps and institutional affiliations.

\section{Author details}

${ }^{1}$ School of Sport and Exercise, Massey University, Private Bag 11-222, Palmerston North, New Zealand. ${ }^{2}$ Massey Institute of Food Science and Technology, Massey University, Palmerston North, New Zealand.

\section{Received: 3 September 2016 Accepted: 14 April 2017} Published online: 27 April 2017

\section{References}

1. Powers S, Nelson W, Hudson M. Exercise-induced oxidative stress in humans: cause and consequences. Free Radic Biol Med. 2011;51(5):942-50.

2. Smith J. Exercise, training and red blood cell turnover. Sports Med. 1995;19(1):9-31.

3. Liu J, et al. Chronically and acutely exercised rats: biomarkers of oxidative stress and endogenous antioxidants. J Appl Physiol. 2000;89(1):21-8.

4. Kennedy $\mathrm{G}$, et al. Oxidative stress levels are raised in chronic fatigue syndrome and are associated with clinical symptoms. Free Radic Biol Med. 2005;39(5):584-9.

5. Lehmann $M$, et al. Training-overtraining: influence of a defined increase in training volume vs training intensity on performance, catecholamines and some metabolic parameters in experienced middle-and long-distance runners. Eur J Appl Physiol Occup Physiol. 1992;64(2):169-77.

6. Xiao W, Chen P, Dong J. Effects of overtraining on skeletal muscle growth and gene expression. Int J Sports Med. 2012;33(10):846-53.

7. Vider J, et al. Acute immune response in respect to exercise-induced oxidative stress. Pathophysiology. 2001;7(4):263-70.

8. Gibala M, et al. Tricarboxylic acid cycle intermediate pool size and estimated cycle flux in human muscle during exercise. Am J Physiol Endocrinol Metab. 1998;275(2):235-42.

9. Dalle-Donne I, et al. Protein carbonyl groups as biomarkers of oxidative stress. Clin Chim Acta. 2003;329(1):23-38.

10. Tsai $K$, et al. Oxidative DNA damage in human peripheral leukocytes induced by massive aerobic exercise. Free Radic Biol Med. 2001;31(11): 1465-72.

11. Mateos R, et al. Determination of malondialdehyde (MDA) by highperformance liquid chromatography in serum and liver as a biomarker for oxidative stress: application to a rat model for hypercholesterolemia and evaluation of the effect of diets rich in phenolic antioxidants from fruits. J Chromatogr B. 2005;827(1):76-82.

12. Mattson MP. Hormesis defined. Ageing Res Rev. 2008;7(1):1-7.

13. Criswell $D$, et al. High intensity training-induced changes in skeletal muscle antioxidant enzyme activity. Med Sci Sports Exerc. 1993;25(10):1135-40.

14. Hollander J, et al. Superoxide dismutase gene expression is activated by a single bout of exercise in rat skeletal muscle. Pfluegers Archiv. 2001;442(3):426-34.

15. Higuchi $M$, et al. Superoxide dismutase and catalase in skeletal muscle: adaptive response to exercise. J Gerontol. 1985;40(3):281-6.

16. Powers $\mathrm{S}$, et al. Influence of exercise and fiber type on antioxidant enzyme activity in rat skeletal muscle. Am J Phys Regul Integr Comp Phys. 1994; 266(2):375-80.

17. Leeuwenburgh $C$, et al. Aging and exercise training in skeletal muscle: responses of glutathione and antioxidant enzyme systems. Am J Phys Regul Integr Comp Phys. 1994;267(2):439-45.

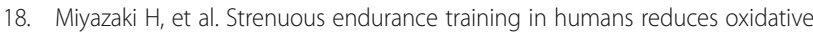
stress following exhausting exercise. Eur J Appl Physiol. 2001;84(1-2):1-6.

19. Steinbacher P, Eckl P. Impact of oxidative stress on exercising skeletal muscle. Biomolecules. 2015;5(2):356-77.

20. Hoogerwerf $A$, Hoitink $A$. The influence of vitamin $C$ administration on the mechanical efficiency of the human organism. Int Z Angew Physiol. 1963;20(2):164-72

21. Keren $\mathrm{G}$, Epstein $\mathrm{Y}$. The effect of high dosage vitamin $\mathrm{C}$ intake on aerobic and anaerobic capacity. J Sports Med Phys Fitness. 1980;20(2):145-8.

22. Keith $R$, Merrill $E$. The effects of vitamin $C$ on maximum grip strength and muscular endurance. J Sports Med Phys Fitness. 1983:23(3):253-6.

23. Sharman I, Down M, Sen R. The effects of vitamin $E$ and training on physiological function and athletic performance in adolescent swimmers. Br J Nutr. 1971;26(02):265-76.
24. Roberts $L$, et al. Vitamin $C$ consumption does not impair training-induced improvements in exercise performance. Int J Sports Physiol Perform. 2011;6(1):58-69.

25. Romano-Ely B, et al. Effect of an isocaloric carbohydrate-protein-antioxidant drink on cycling performance. Med Sci Sports Exerc. 2006;38(9):1608-16.

26. Yfanti $C$, et al. Antioxidant supplementation does not alter endurance training adaptation. Med Sci Sports Exerc. 2010;42(7):1388-95.

27. Akova B, et al. Exercise-induced oxidative stress and muscle performance in healthy women: role of vitamin E supplementation and endogenous oestradiol. Eur J Appl Physiol. 2001;84(1-2):141-7.

28. Shephard $R$, et al. Vitamin $E$, exercise, and the recovery from physical activity. Eur J Appl Physiol Occup Physiol. 1974;33(2):119-26.

29. Bryer S, Goldfarb A. Effect of high dose vitamin C supplementation on muscle soreness, damage, function, and oxidative stress to eccentric exercise. Int J Sport Nutr Exerc Metab. 2006;16(3):270-80.

30. Thompson D, et al. Prolonged vitamin C supplementation and recovery from demanding exercise. Int J Sport Nutr Exerc Metab. 2001;11(4):466-81.

31. Gomez-Cabrera M, et al. Oral administration of vitamin C decreases muscle mitochondrial biogenesis and hampers training-induced adaptations in endurance performance. Am J Clin Nutr. 2008;87(1):142-9.

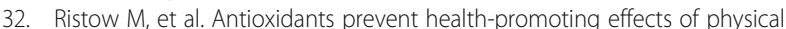
exercise in humans. Proc Natl Acad Sci. 2009;106(21):8665-70.

33. Zhang $\mathrm{H}$, Forman $\mathrm{H}$, Choi J. Gamma-glutamyl transpeptidase in glutathione biosynthesis. Methods Enzymol. 2005;401:468-83.

34. Go Y-M, Jones D. Cysteine/cystine redox signaling in cardiovascular disease. Free Radic Biol Med. 2011;50(4):495-509.

35. Buzina R, Suboticanec K. Vitamin C and physical working capacity. Int J Vitam Nutr Res. 1985:27:157.

36. Fischer $C$, et al. Supplementation with vitamins $C$ and $E$ inhibits the release of interleukin-6 from contracting human skeletal muscle. J Physiol. 2004; 558(2):633-45.

37. Simon-Schnass I, Pabst H. Influence of vitamin E on physical performance. Int J Vitam Nutr Res. 1987;58(1):49-54.

38. Wadley G, McConell G. High-dose antioxidant vitamin C supplementation does not prevent acute exercise-induced increases in markers of skeletal muscle mitochondrial biogenesis in rats. J Appl Physiol. 2010;108(6):1719-26.

39. Satoshi S, et al. Exercise-induced lipid peroxidation and leakage of enzymes before and after vitamin E supplementation. Int J Biochem. 1989;21(8):835-8.

40. Lawrence J, et al. Effects of alpha-tocopherol acetate on the swimming endurance of trained swimmers. Am J Clin Nutr. 1975;28(3):205-8.

41. Nieman D, et al. Influence of vitamin C supplementation on oxidative and immune changes after an ultramarathon. J Appl Physiol. 2002;92(5):1970-7.

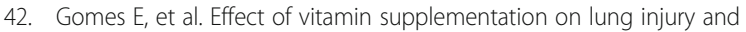
running performance in a hot, humid, and ozone-polluted environment. Scand J Med Sci Sports. 2011:21(6):452-60.

43. Close $\mathrm{G}$, et al. Ascorbic acid supplementation does not attenuate postexercise muscle soreness following muscle-damaging exercise but may delay the recovery process. Br J Nutr. 2006;95(5):976-81.

44. Howatson $\mathrm{G}$, et al. Influence of tart cherry juice on indices of recovery following marathon running. Scand J Med Sci Sports. 2010;20(6):843-52.

45. Bailey $D$, et al. Oxidative stress, inflammation and recovery of muscle function after damaging exercise: effect of 6-week mixed antioxidant supplementation. Eur J Appl Physiol. 2011:111(6):925-36.

46. Avery $\mathrm{N}$, et al. Effects of vitamin $\mathrm{E}$ supplementation on recovery from repeated bouts of resistance exercise. J Strength Cond Res. 2003:17(4):801-9.

47. Jakemanl P, Maxwell S. Effect of antioxidant vitamin supplementation on muscle function after eccentric exercise. Eur J Appl Physiol Occup Physiol. 1993;67(5):426-30

48. Connolly D, McHugh M, Padilla-Zakour O. Efficacy of a tart cherry juice blend in preventing the symptoms of muscle damage. Br J Sports Med. 2006;40(8):679-83.

49. McLeay $Y$, et al. Effect of New Zealand blueberry consumption on recovery from eccentric exercise-induced muscle damage. J Int Soc Sports Nutr. 2012;9(1):19.

50. Paulsen $\mathrm{G}$, et al. Vitamin $\mathrm{C}$ and $\mathrm{E}$ supplementation hampers cellular adaptation to endurance training in humans: a double-blind, randomised, controlled trial. J Physiology. 2014;592(8):1887-901.

51. Paulsen $\mathrm{G}$, et al. Vitamin $\mathrm{C}$ and $\mathrm{E}$ supplementation alters protein signalling after a strength training session, but not muscle growth during 10 weeks of training. J Physiol. 2014;592(24):5391-408.

52. Gomez-Cabrera M, Ristow M, Viña J. Antioxidant supplements in exercise: worse than useless? Am J Physiol Endocrinol Metab. 2012;302(4):476-7. 
53. Fang $Y$, Yang $S$, Wu G. Free radicals, antioxidants, and nutrition. Nutrition. 2002;18(10):872-9.

54. Powers S, Jackson M. Exercise-induced oxidative stress: cellular mechanisms and impact on muscle force production. Physiol Rev. 2008;88(4):1243-76.

55. Halliwell B. Commentary: vitamin C: antioxidant or pro-oxidant in vivo? Free Radic Res. 1996;25(5):439-54.

56. Cillard J, et al. a-Tocopherol prooxidant effect in aqueous media: increased autoxidation rate of linoleic acid. J Am Oil Chem Soc. 1980;57(8):252-5.

57. Childs $\mathrm{A}$, et al. Supplementation with vitamin $\mathrm{C}$ and $\mathrm{N}$-acetyl-cysteine increases oxidative stress in humans after an acute muscle injury induced by eccentric exercise. Free Radic Biol Med. 2001;31(6):745-53.

58. Knez W, Jenkins D, Coombes J. Oxidative stress in half and full Ironman triathletes. Med Sci Sports Exerc Sci. 2007;39(2):283-8.

59. Yordi E et al. Antioxidant and pro-oxidant effects of polyphenolic compounds and structure-activity relationship evidence. Nutrition, WellBeing and Health. Croatia: InTech; 2012: p. 23-48

60. Hayes J, Mclellan L. Glutathione and glutathione-dependent enzymes represent a co-ordinately regulated defence against oxidative stress. Free Radic Res. 1999;31(4):273-300.

61. Jones D. Redox potential of GSH/GSSG couple: Assay and biological significance. Methods Enzymol. 2002;348:93-112.

62. Herzenberg $L$, et al. Glutathione deficiency is associated with impaired survival in HIV disease. Proc Natl Acad Sci. 1997:94(5):1967-72.

63. Holmay M, et al. N-acetylcysteine boosts brain and blood glutathione in gaucher and Parkinson's diseases. Clin Neuropharmacol. 2013;36(4):103-6.

64. Bridgeman $\mathrm{M}$, et al. Cysteine and glutathione concentrations in plasma and bronchoalveolar lavage fluid after treatment with $\mathrm{N}$-acetylcysteine. Thorax. 1991:46(1):39-42.

65. Medved I, et al. N-acetylcysteine enhances muscle cysteine and glutathione availability and attenuates fatigue during prolonged exercise in endurancetrained individuals. J Appl Physiol. 2004;97(4):1477-85.

66. Michailidis $Y$, et al. Thiol-based antioxidant supplementation alters human skeletal muscle signaling and attenuates its inflammatory response and recovery after intense eccentric exercise. Am J Clin Nutr. 2013;98(1):233-45.

67. Wang $\mathrm{S}$, et al. Methionine and cysteine affect glutathione level, glutathionerelated enzyme activities and the expression of glutathione S-transferase isozymes in rat hepatocytes. J Nutr. 1997;127(11):2135-41.

68. Terrill J, et al. Increasing taurine intake and taurine synthesis improves skeletal muscle function in the mdx mouse model for Duchenne Muscular Dystrophy. J Physiol. 2016:594(11):3095-110.

69. Duarte T, Lunec J. Review: when is an antioxidant not an antioxidant? a review of novel actions and reactions of vitamin C. Free Radic Res. 2005; 39(7):671-86.

70. Kerksick C, Willoughby D. The antioxidant role of glutathione and N-acetylcysteine supplements and exercise-induced oxidative stress. J Int Soc Sports Nutr. 2005;2(2):1.

71. Silva $\mathrm{L}$, et al. $\mathrm{N}$-acetylcysteine supplementation and oxidative damage and inflammatory response after eccentric exercise. Int J Sport Nutr. 2008;18(4): 379-88.

72. Cobley J, et al. N-Acetylcysteine's attenuation of fatigue after repeated bouts of intermittent exercise: practical implications for tournament situations. Int J Sport Nutr Exerc Metab. 2011;21(6):451-61.

73. McKenna MJ, et al. $\mathrm{N}$-acetylcysteine attenuates the decline in muscle $\mathrm{Na}+$, $\mathrm{K}+$-pump activity and delays fatigue during prolonged exercise in humans. J Physiol. 2006;576(1):279-88.

74. Hayden P, Stevens J. Cysteine conjugate toxicity, metabolism, and binding to macromolecules in isolated rat kidney mitochondria. Mol Pharmacol. 1990;37(3):468-76.

75. Karlsen R, et al. Morphological changes in rat brain induced byl-cysteine injection in newborn animals. Brain Res. 1981;208(1):167-80.

76. Dilger $\mathrm{R}$, et al. Excess dietary $\mathrm{L}$-cysteine, but not $\mathrm{L}$-cystine, is lethal for chicks but not for rats or pigs. J Nutr. 2007;137(2):331-8.

77. Whillier $\mathrm{S}$, et al. Role of $\mathrm{N}$-acetylcysteine and cystine in glutathione synthesis in human erythrocytes. Redox Rep. 2009;14(3):115-24.

78. Meister A. Glutathione deficiency produced by inhibition of its synthesis, and its reversal; applications in research and therapy. Pharmacol Ther. 1991;51(2):155-94.

79. Yatabe $Y$, et al. Effects of taurine administration in rat skeletal muscles on exercise. J Orthop Sci. 2003;8(3):415-9.

80. Zhang $M$, et al. Role of taurine supplementation to prevent exerciseinduced oxidative stress in healthy young men. Amino Acids. 2004;26(2): 203-7.
81. Miyazaki T, et al. Optimal and effective oral dose of taurine to prolong exercise performance in rat. Amino Acids. 2004;27(3-4):291-8.

82. Supinski $\mathrm{G}$, et al. $\mathrm{N}$-acetylcysteine administration alters the response to inspiratory loading in oxygen-supplemented rats. J Appl Physiol. 1997;82(4):1119-25.

83. Reid $\mathrm{M}$, et al. $\mathrm{N}$-acetylcysteine inhibits muscle fatigue in humans. J Clin Investig. 1994;94(6):2468-74.

84. Matuszczak $\mathrm{Y}$, et al. Effects of $\mathrm{N}$-acetylcysteine on glutathione oxidation and fatigue during handgrip exercise. Muscle Nerve. 2005;32(5):633-8.

85. Schaffer SW, et al. Physiological roles of taurine in heart and muscle. J Biomed Sci. 2010;17 Suppl 1:S2.

86. Goodman C, et al. Taurine supplementation increases skeletal muscle force production and protects muscle function during and after high-frequency in vitro stimulation. J Appl Physiol. 2009;107(1):144-54.

87. Hardison W. Hepatic taurine concentration and dietary taurine as regulators of bile acid conjugation with taurine. Gastroenterology. 1978;75(1):71-5.

88. Uozumi Y, et al. Myogenic differentiation induces taurine transporter in association with taurine-mediated cytoprotection in skeletal muscles. Biochem J. 2006;394(3):699-706.

89. Balkan J, et al. Taurine has a protective effect against thioacetamideinduced liver cirrhosis by decreasing oxidative stress. Hum Exp Toxicol. 2001;20(5):251-4.

90. Geiss $\mathrm{K}$, et al. The effect of a taurine-containing drink on performance in 10 endurance-athletes. Amino Acids. 1994;7(1):45-56.

91. Alford $\mathrm{C}, \mathrm{Cox} H$, Wescott R. The effects of red bull energy drink on human performance and mood. Amino Acids. 2001;21(2):139-50.

92. Imagawa T, et al. Caffeine and taurine enhance endurance performance. Int J Sports Med. 2009;30(07):485-8.

93. Balshaw $\mathrm{T}$, et al. The effect of acute taurine ingestion on $3-\mathrm{km}$ running performance in trained middle-distance runners. Amino Acids. 2013;44(2): $555-61$.

94. Rutherford J, Spriet L, Stellingwerff T. The effect of acute taurine ingestion on endurance performance and metabolism in well-trained cyclists. Int J Sport Nutr. 2010;20(4):322-9.

95. Powers $\mathrm{S}$, et al. Reactive oxygen species are signalling molecules for skeletal muscle adaptation. Exp Physiol. 2010;95(1):1-9.

96. Akimoto T, et al. Exercise stimulates Pgc-1a transcription in skeletal muscle through activation of the p38 MAPK pathway. J Biol Chem. 2005;280(20): 19587-93.

97. Li Y-P, Reid MB. Effect of tumor necrosis factor-a on skeletal muscle metabolism. Curr Opin Rheumatol. 2001;13(6):483-7.

98. Irrcher I, et al. Regulation of mitochondrial biogenesis in muscle by endurance exercise. Sports Med. 2003;33(11):783-93.

99. Wilkinson $\mathrm{SB}$, et al. Differential effects of resistance and endurance exercise in the fed state on signalling molecule phosphorylation and protein synthesis in human muscle. J Physiol. 2008;586(15):3701-17.

100. Evelo C, et al. Changes in blood glutathione concentrations, and in erythrocyte glutathione reductase and glutathione S-transferase activity after running training and after participation in contests. Eur J Appl Physiol Occup Physiol. 1992;64(4):354-8.

101. Marzatico F, et al. Blood free radical antioxidant enzymes and lipid peroxides following long-distance and lactacidemic performances in highly trained aerobic and sprint athletes. J Sports Med Phys Fitness. 1997;37(4): 235-9.

102. Atherton $\mathrm{P}$, Smith K. Muscle protein synthesis in response to nutrition and exercise. J Physiol. 2012;590(5):1049-57.

103. Slattery KM, et al. Effect of $\mathrm{N}$-acetylcysteine on cycling performance after intensified training. Med Sci Sports Exerc. 2014;46(6):1114-23.

104. Leelarungrayub $\mathrm{D}$, et al. $\mathrm{N}$-acetylcysteine supplementation controls total antioxidant capacity, creatine kinase, lactate, and tumor necrotic factoralpha against oxidative stress induced by graded exercise in sedentary men. Oxidative Med Cell Longev. 2011;2011:329643.

105. Trewin AJ, et al. Effect of $\mathrm{N}$-acetylcysteine infusion on exercise-induced modulation of insulin sensitivity and signaling pathways in human skeletal muscle. Am J Physiol Endocrinol Metab. 2015;309(4):E388-97.

106. Petersen $\mathrm{A}$, et al. Infusion with the antioxidant $\mathrm{N}$-acetylcysteine attenuates early adaptive responses to exercise in human skeletal muscle. Acta Physiol. 2012;204(3):382-92.

107. Özkan Y, Özkan E, Şimşek B. Plasma total homocysteine and cysteine levels as cardiovascular risk factors in coronary heart disease. Int J Cardiol. 2002;82(3):269-77. 
108. Kleinveld H, Demacker $\mathrm{P}$, Stalenhoef A. Failure of $\mathrm{N}$-acetylcysteine to reduce low-density lipoprotein oxidizability in healthy subjects. Eur J Clin Pharmacol. 1992:43(6):639-42.

109. Oikawa S, et al. N-acetylcysteine, a cancer chemopreventive agent, causes oxidative damage to cellular and isolated DNA. Carcinogenesis. 1999;20(8): 1485-90.

110. Stey $\mathrm{C}$, et al. The effect of oral N-acetylcysteine in chronic bronchitis: a quantitative systematic review. Eur Respir J. 2000;16(2):253-62.

111. Dawson Jr R, et al. The cytoprotective role of taurine in exercise-induced muscle injury. Amino Acids. 2002;22(4):309-24

112. Ito $T$, et al. Tissue depletion of taurine accelerates skeletal muscle senescence and leads to early death in mice. PLoS One. 2014;9(9):e107409.

113. da Silva $L$, et al. Effects of taurine supplementation following eccentric exercise in young adults. Appl Physiol Nutr Metab. 2013;39(1):101-4.

114. Silva LA, et al. Taurine supplementation decreases oxidative stress in skeletal muscle after eccentric exercise. Cell Biochem Funct. 2011;29(1):43-9.

115. Badaloo A, et al. Cysteine supplementation improves the erythrocyte glutathione synthesis rate in children with severe edematous malnutrition. Am J Clin Nutr. 2002;76(3):646-52.

116. Atkuri K, Mantovani J, Herzenberg L. N-Acetylcysteine-a safe antidote for cysteine/glutathione deficiency. Curr Opin Pharmacol. 2007;7(4):355-9.

117. Wald DS, Law M, Morris JK. Homocysteine and cardiovascular disease: evidence on causality from a meta-analysis. BMJ. 2002;325(7374):1202.

118. Nestoros J, Ban T, Lehmann H. Transmethylation hypothesis of schizophrenia: methionine and nicotinic acid. Int Pharmacopsychiatry. 1976; 12(4):215-46.

119. Houltham S, Stark C, Stannard S. Two week Keratin-based protein supplementation is comparable in gastrointestinal milk handling to a milkbased equivalent. J Hum Nutr Food Sci. 2014;2(5):1047.

120. Wolber FM, et al. Cysteic acid in dietary keratin is metabolized to glutathione and liver taurine in a rat model of human digestion. Nutrients. 2016;8(2):104.

\section{Submit your next manuscript to BioMed Central and we will help you at every step:}

- We accept pre-submission inquiries

- Our selector tool helps you to find the most relevant journal

- We provide round the clock customer support

- Convenient online submission

- Thorough peer review

- Inclusion in PubMed and all major indexing services

- Maximum visibility for your research

Submit your manuscript at www.biomedcentral.com/submit 